04

\title{
Подавление потоков убегающих электронов и особенности пробоя рабочего газа в торсатроне Ураган-2М
}

\author{
() В.Б. Коровин, И.К. Тарасов, Е.Д. Крамской, Д.А. Ситников, Н.Б. Древаль, А.В. Лозин, М.М. Козуля \\ Институт ффизики плазмы ННЦ „Харьковский фризико-технический институт“, \\ 61108 Харьков, Украина \\ e-mail: korovin@kipt.kharkov.ua
}

(Поступило в Редакцию 19 июня 2017 г.)

\begin{abstract}
Приведены результаты экспериментального исследования влияния убегающих электронов на параметры пробоя нейтрального газа и подавления потока убегающих электронов в торсатроне Ураган-2М. В процессе проведения экспериментов методом сравнительного анализа исследовалось влияние на временные характеристики пробоя наличие этого потока или его отсутствие. Показано, что при наличии потока убегающих электронов пробой происходит в среднем на $1.5 \mathrm{~ms}$ раньше в сравнении с пробоем при его отсутствии. Это подтверждают результаты, полученные ранее на торсатроне Ураган-3М, что позволяет продолжить описание некоторых аспектов динамики убегающих электронов, ее закономерностей и представить ряд рекомендаций по управлению потоками таких электронов в установках термоядерного синтеза.
\end{abstract}

DOI: 10.21883/JTF.2018.07.46164.2388

\section{Введение}

Одной из задач физики термоядерного синтеза является исследование влияния потоков дрейфующих частиц на устойчивость плазменного шнура и на параметры плазмы. К факторам, которые обусловливают формирование неамбиполярных потоков в объеме удержания, можно отнести и динамику изменения напряженности магнитного поля во времени. В малых тороидальных установках термоядерного синтеза магнитное поле не является стационарным, а генерируется в импульсном режиме. За счет резкого изменения напряженности магнитного поля на фронтах импульса происходит формирование вихревого электрического поля, которое ориентировано в тороидальном направлении. В результате воздействия такого поля в объеме удержания могут формироваться потоки так называемых убегающих электронов (УЭ). Здесь речь идет об ускоренных в тороидальном электрическом поле электронах до энергий, при которых упругими столкновениями с другими частицами можно пренебречь.

Наличие потоков УЭ в объеме удержания во время разряда оказывает значительное влияние как на устойчивость плазменного шнура, так и на энергетический баланс системы [1]. В частности, прохождение потока ускоренных заряженных частиц в плазменном шнуре приводит к дополнительному нагреву плазмы, a также к возрастанию ее плотности. Однако при взаимодействии таких быстрых частиц с плазмой может развиваться ряд неустойчивостей. Поэтому УЭ представляют собой одну из основных угроз в современных термоядерных установках. Помимо этого существует возможность повреждения первой стенки таких установок при попадании на нее мощного потока высокоэнергичных частиц. На сегодняшний день существует основная модель возникновения убегающих электронов, предложенная Драйсером. Условия убегания возникают, если приложенное электрическое поле будет больше, чем величина $E_{D}$, определяемая из выражения $E_{D}=n_{e} e^{3} \ln \Lambda / 4 \pi \varepsilon_{0}^{2} T_{e}$, где $n_{e}, e$ и $T_{e}$ соответственно плотность, абсолютная величина заряда и температура электронов плазмы, а $\ln \Lambda-$ кулоновский логарифм, при этом тепловые электроны начинают ,убегать“. Поле $E_{D}$ называется в честь первооткрывателя убегающих электронов Драйсера (Dreicer) — драйсеровским полем [2].

В предыдущих работах рассматривалась принципиальная возможность подавления потока убегающих электронов при помощи подачи потенциала на антенну ВЧ генератора, находящуюся внутри камеры торсатрона. Подавление УЭ было достигнуто при подаче как отрицательного, так и положительного потенциала на антенну [3,4].

Математическое моделирование эффекта подавления потока убегающих электронов электростатическим потенциалом проводилось в работе [3]. В ней показано, что при движении электрона в вихревом электрическом и электростатическом полях его потенциальная энергия падает в положительном направлении продольной координаты, и электрон движется в этом направлении, постоянно увеличивая свою кинетическую энергию. При наложении электростатического подавляющего потенциала достаточно большой величины, пространственная зависимость эффективной потенциальной энергии электрона становится немонотонной. При наложении положительного подавляющего потенциала она имеет минимум вблизи максимума подавляющего потенциала и потенциальную яму в его окрестности, а также максимум справа от нее. Если электрон родился внутри такой потенциальной ямы, и его эффективная потенциальная энергия меньше, чем $P_{\max }-K_{0}\left(P_{\max }-\right.$ максимальное значение эффективной потенциальной энергии, $K_{0}-$ 
начальная кинетическая энергия электрона), то он не может вылететь из ямы, отражаясь от точек, где $K=0$. Он не получает энергию от вихревого электрического поля и не участвует в процессе лавинообразования. Популяцию ускоренных электронов пополняют только электроны, которые родились вне потенциальной ямы. В случае же наложения отрицательного подавляющего потенциала обширная потенциальная яма образуется вне области наложения электростатического потенциала. В этом случае количество электронов, которые могут пополнять лавинный процесс, значительно меньше, чем в случае наложения положительного подавляющего потенциала. Поэтому в дальнейших экспериментах использовался подавляющий потенциал отрицательной полярности. В предыдущих экспериментах антенна, на которую подавался подавляющий потенциал, была пассивной, т.е. ВЧ мощность в нее не вводилась. Плазма же создавалась другой антенной, запитанной от второго ВЧ генератора. Обычно при проведении исследований на торсатронах Ураган-2М и Ураган-3М используются две антенны, питающиеся от двух ВЧ генераторов [5]. $\mathrm{C}$ помощью одной из них создается плазма в рабочей камере торсатрона, а посредством ВЧ мощности, вводимой во вторую антенну, осуществляется нагрев плазменного шнура. Для того чтобы не сооружать специальную конструкцию для ввода в вакуумную камеру дополнительного электрода, предназначенного для подавления потока УЭ, было решено изучить возможность использования одной из работающих ВЧ антенн в качестве электрода для подавления УЭ. Для экспериментальной проверки этого вопроса была выбрана рамочная антенна, с помощью которой создавался начальный пробой рабочего газа.

\section{Постановка эксперимента}

В торсатроне Ураган-2М проводились эксперименты по ВЧ нагреву плазмы на частоте ионно-циклотронного резонанса в атмосфере водорода. Плазма создавалась ВЧ генератором Каскад-1, работающим на частоте $5.6 \mathrm{MHz}$, нагрев плазмы осуществлялся ВЧ мощностью от второго генератора Каскад-2, работающего на частоте 5.2 MHz. Схема ВЧ нагрева такова: ВЧ энергия от генератора через линию передачи поступает в антенну через устройство согласования, которое состоит из индуктивности $L_{m}$ и емкости $C_{m}$ (рис. 1$)$. В начальных экспериментах давление водорода в камере составляло $6-8 \cdot 10^{-6}$ Torr, a величина магнитного поля при этом равнялась $3.6 \mathrm{kOe}$. Величина ВЧ мощности, вводимой в антенну коленвального типа от генератора Каскад-1, составляла порядка $80 \mathrm{~kW}$, а вводимая ВЧ мощность в антенну рамочного типа от генератора Каскад-2 порядка $100 \mathrm{~kW}$. Для подавления потока УЭ на антенну, с помощью которой осуществлялся ВЧ пробой, подавалось также и напряжение подавления - $U_{s}$ (рис. 1). Причем, в одном случае, это напряжение было неизменным (постоянным) в течение всего импульса магнитного поля. В другом случае подавался импульс отрицательного напряжения подавления. Длительность этого импульса подавления изменялась в пределах от $10 \mathrm{~ms}$ до $1 \mathrm{~s}$. Также изменялся и момент его подачи по отношению к импульсу магнитного поля - от начала роста тока в обмотках магнитной системы установки до выхода на стационарный режим этого тока.

Чтобы защитить источник управляющего напряжения от воздействия ВЧ напряжения, подаваемого на антенну от генератора, непосредственно к антенным выводам был подсоединен Г-образный фильтр низких частот. Фильтр образован индуктивностью $L_{f}$ и емкостью $C_{f}$ (рис. 1). Его полоса пропускания рассчитывалась по формуле $\Delta \omega=1 / \sqrt{L C}$ и по соображениям безопасности должна была составлять менее $100 \mathrm{kHz}$. Такая узкая полоса фильтра была выбрана, чтобы исключить воздействие, в том числе и разностных частот ВЧ генераторов. При использовании конкретных установленных элементов $\left(C_{f}=10000 \mathrm{pF}, L_{f}=6 \mathrm{mH}\right)$ полоса пропускания фильтра составила $20.5 \mathrm{kHz}$, что практически исключает возможность воздействия на источник подавляющего напряжения практически всех комбинационных частот, возникающих в камере установки при создании плазмы.

\section{Результаты}

На первом этапе проводилось исследование возможности подавления самопроизвольно формирующихся потоков ускоренных частиц в торсатроне путем подачи импульсного подавляющего напряжения $U_{s}$ на антеннуэлектрод, с помощью которой осуществлялся пробой рабочего газа. Подавались импульсы отрицательной полярности длительностью $500 \mathrm{~ms}$ и амплитудой от 25 до $50 \mathrm{~V}$. Наличие УЭ контролировалось измерением рентгеновского излучения на фронтах магнитного поля.

Было обнаружено, что при подаче отрицательного импульса напряжения амплитудой $-25 \mathrm{~V}$ подавление потока УЭ не происходило. В то время как при амплитуде импульса $-50 \mathrm{~V}$, было достигнуто уверенное подавление потока УЭ при одинаковой длительности импульсов напряжения подавления $U_{s}$ и других параметров эксперимента.

Следующим шагом была попытка установить необходимый момент времени подачи импульса запирающего напряжения $U_{s}$ по отношению к импульсу тока в магнитных катушках установки и определение минимально достаточной длительности этого импульса. За начальный момент подачи $U_{s}$ была взята задержка от старта импульса магнитного поля, равная $100 \mathrm{~ms}$. В этой точке подавление было достигнуто при длительности импульса подавления, равной $\tau_{i}=1 \mathrm{~s}$. Постепенно уменьшали его длительность и достигли минимального значения $\tau_{i}=10 \mathrm{~ms}$, при котором еще наблюдалось подавление потока УЭ. Затем импульс подавления (постоянной 


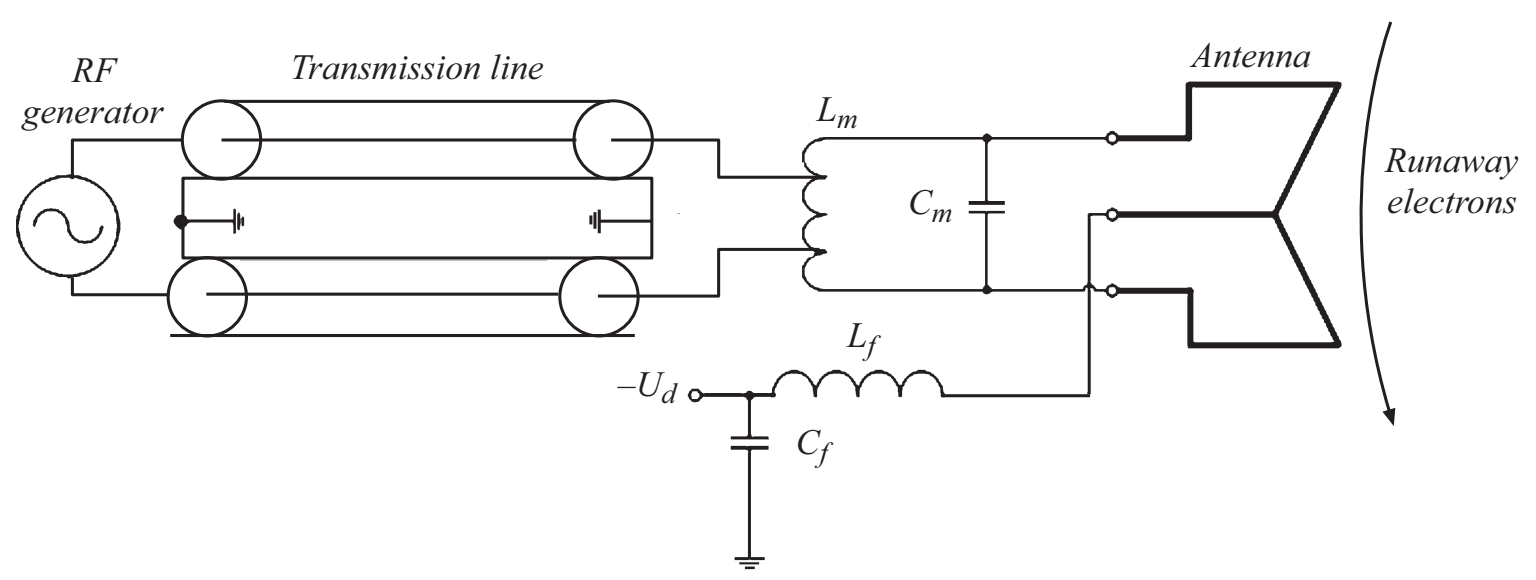

Рис. 1. Принципиальная схема подачи управляющего сигнала для подавления потока УЭ на работающую антенну.

длительности) перемещали во времени по фронту нарастания импульса тока магнитного поля. Эксперименты показали, что подача такого импульса подавляющего напряжения на антенну, до момента включения ВЧ импульса, создающего плазму, позволяет осуществить подавление потока УЭ.

Также было замечено, что длительность импульса не имеет большого значения. Это объясняется наличием в схеме конденсатора фильтра $C_{f}$ (рис. 1), который сначала заряжается напряжением импульса подавления, а затем происходит его разряд уже в момент времени, когда зарождается поток УЭ. Для проверки этого предположения сделали следующее. За $1 \mathrm{~s}$ до старта импульса магнитного поля на антенну, а значит и на конденсатор фильтра, подавали отрицательный импульс постоянного напряжения амплитудой $-50 \mathrm{~V}$. Длительность импульса порядка $10-30 \mathrm{~ms}$. Таким образом был осуществлен заряд конденсатора фильтра $C_{f}$ до уровня напряжения подавления $U_{s}$ перед подачей импульса магнитного поля. Затем источник подавляющего напряжения отключался. И эффект подавления УЭ также был достигнут, но уже путем использования только энергии заряда конденсатора. В этом эксперименте отсутствовали любые иные источники создания потенциала подавления на антенне-электроде, поэтому была исключена возможность влияния их внутреннего сопротивления на процесс подавления УЭ.

После проведения этого этапа экспериментов появилась возможность оценить энергию, необходимую для подавления потока УЭ. Как известно, энергия конденсатора равна $E=C U^{2} / 2$. При напряжении подавления в $50 \mathrm{~V}$ (при котором достигалось подавление) энергия, запасенная конденсатором фильтра, $E_{c}=12.5 \mu \mathrm{J}$, а при напряжении $25 \mathrm{~V}$, при котором подавление не достигалось, $E_{c}=3.12 \mu \mathrm{J}$. Таким образом, можно предположить, что для подавления потока УЭ, возникающего в установке Ураган-2М, необходима энергия подавления, составляющая порядка $10 \mu \mathrm{J}$.

\section{Влияние подавления потока убегающих электронов на параметры плазмы}

Была сделана проверка воздействия механизма подавления УЭ на начальную фазу пробоя рабочего газа. Для этого контролировалась интенсивность излучения в видимом диапазоне от разряда в камере.

На рис. 2 приведены графики интенсивности излучения в видимом диапазоне частот от плазмы в камере установки Ураган-3М. Видно, что при наложении потенциала подавления $U_{s}$ интенсивность излучения уменьшается (на 10-15\%) по сравнению с обычным режимом без подавления. Это может свидетельствовать о влияния эффекта подавления УЭ на разряд рабочего газа в вакуумной камере установки. Для уточнения этого влияния было проведено сравнение интенсивности излучения спектральной линии водорода $H_{\alpha}$ (длина волны $656.285 \mathrm{~nm}$ ) и рентгеновского излучения плазмы при наличии потока УЭ и в его отсутствие. Измерения проводились детектором с интерференционным фильтром, расположенным перпендикулярно плазменному

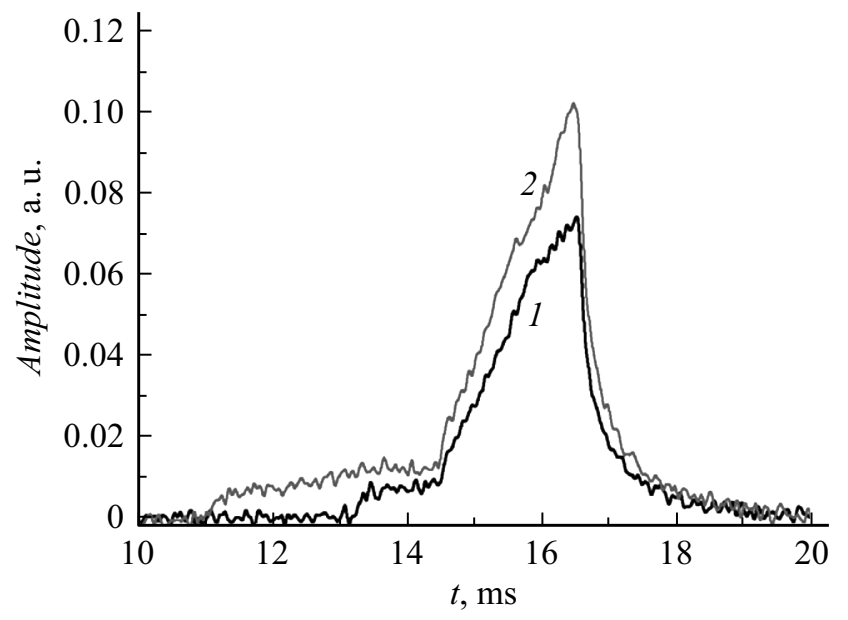

Рис. 2. Излучение видимого света от разряда в камере: 1 с включенным потенциалом подавления, 2 - без подавления. 
шнуру. При сравнении интенсивности излучения линии водорода $H_{\alpha}(656.285 \mathrm{~nm})$ заметна задержка сигнала излучения линии водорода $H_{\alpha}$ при включенном потенциале подавления примерно на $1 \mathrm{~ms}$, при этом уровень сигнала $H_{\alpha}$ при подавлении несколько выше (рис. 3).

Рис. 4 иллюстрирует влияние потока УЭ на процессы в плазме. На нем видно, что сигнал мягкого рентгеновского излучения из плазмы, который косвенно можно связать с ее температурой, при наличии потока УЭ примерно в 2 раза выше, чем в случае подавления этого потока.

Было также проведено изучение влияния напряжения подавления $U_{s}$ УЭ на время пробоя рабочего газа (времени задержки холостого хода $\left(t_{o-s}\right)$ ВЧ генератора) (рис. 5). Дальше говорим о времени холостого хода ВЧ генератора (промежуток времени от момента подачи ВЧ напряжения на антенну до момента пробоя газа), потому что этот параметр очень важен

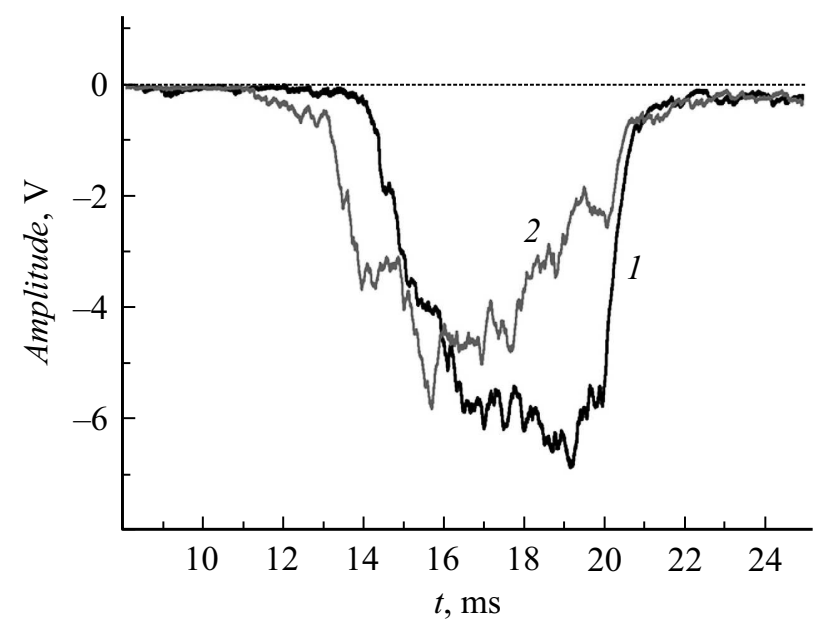

Рис. 3. Интенсивность излучения спектральной линии водорода $H_{\alpha}(656.285 \mathrm{~nm}): 1-$ с включенным потенциалом подавления, 2 - без подавления.

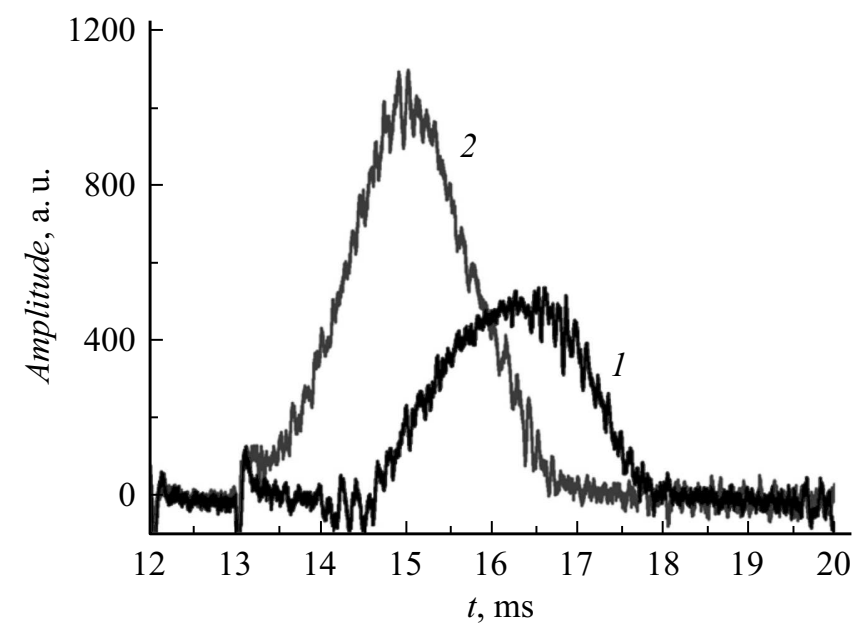

Рис. 4. Интенсивности излучения мягкого рентгена: 1 с включенным потенциалом подавления, 2 - без подавления.

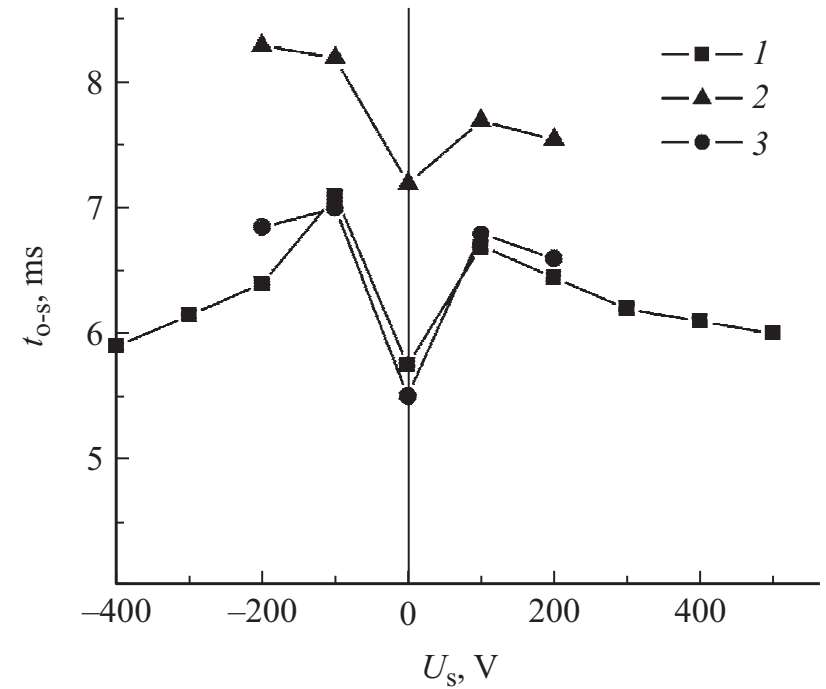

Рис. 5. Зависимость времени пробоя рабочего газа (задержки холостого хода $-t_{o-s}$ ВЧ генератора) от величины приложенного потенциала подавления и от давления в рабочей камере, $P: 1-7.2 \cdot 10^{-4}, 2-8.3 \cdot 10^{-4}, 3-5.3 \cdot 10^{-4} \mathrm{~Pa}$.

для обеспечения безопасной работы всего ВЧ силового тракта. Так как в отсутствие пробоя рабочего газа все силовые элементы, включая элементы контура ВЧ генератора, линия передачи, согласующее устройство и антенные выводы, находятся под высоким ВЧ напряжением. А в линии передачи возникает стоячая волна с большой амплитудой. Эти факторы могут привести к возникновению ВЧ пробоев и выходу оборудования из строя. Было обнаружено, что при осуществлении подачи на антенну-электрод напряжения подавления $U_{s}$, равного $100 \mathrm{~V}$, происходит увеличение времени задержки создания плазмы $t_{o-s}-$ времени холостого хода ВЧ генератора. При дальнейшем увеличении $U_{s}$ до $500 \mathrm{~V}$, происходит постепенное уменьшение этой задержки. Причем видно, что зависимость времени задержки пробоя рабочего газа практически симметрична при изменении полярности напряжения подавления. Конечно, на время холостого хода влияет также и давление рабочего газа в камере. Но характер поведения кривых при разных значениях давления практически одинаков.

Таким образом, экспериментально доказана возможность осуществления подавления УЭ в камере торсатрона, используя в качестве подавляющего электрода ВЧ антенну, в которую вводится ВЧ мощность для создания плазмы. Обнаружена зависимость времени задержки пробоя рабочего газа (холостого хода ВЧ генератора) от уровня подавляющего потенциала $U_{s}$.

\section{Список литературы}

[1] Vlasenkov V.S. et. al. // Nucl. Fusion. 1973. Vol. 13. P. 509-516.

[2] Dreicer H. // Phys. Rev. 1959. Vol. 115. P. 238-248. 
[3] Моисеенко В.Е., Коровин В.Б., Тарасов И.К., Тарасов М.И., Ситников Д.А., Гаркуша И.Е., Заманов Н.В., Лытова М.А., Павличенко Р.О., Кулага А.Е., Пашнев В.К. // Письма в ЖТФ. 2014. Т. 40. Вып 15. С. 80-87.

[4] Moiseenko V.E., Tarasov I.K., Tarasov M.I., Sitnikov D.A., Korovin V.B., Komarov A.D., Kozachek A.S., Krupnik L.I., Zhezhera A.I., Pavlichenko R.O., Zamanov N.V., Kulaga A.Ye., Shapoval A.N., Lytova M.A., Maznichenko S.M., Solodovchenko S.I., Shtan A.F. // Problems of Atomic Science and Technology. Series „Plasma Physics“. 2014. N 6. P. 266-268.

[5] Коровин В.Б., Крамской Е.Д. // Problems of Atomic Science and Technology. Series „Plasma Physics“. 2012. N 6 (82). P. 19-21. 